\title{
Dynamic Changes in Glottal Resistance during Exposure to Severe Hypoxia in Neonatal Rats In Situ
}

\author{
MATHIAS DUTSCHMANN AND JULIAN F.R. PATON \\ Department of Physiology [M.D.], University of Göttingen, 37076 Göttingen, Germany; and Department of \\ Physiology [J.F.R.P.], School of Medical Sciences, University of Bristol, Bristol BS8 1TD, United Kingdom
}

\begin{abstract}
The neural control of respiratory airflow via the vocal fold is characterized by inspiratory abduction and postinspiratory (early expiratory) adduction causing decreases and increases in glottal resistance, respectively. The postinspiratory increase in glottal resistance plays a major role in braking the speed of expiratory airflow, to act against the high recoil pressure of the neonatal rat lung. In the present study, we investigated changes in upper airway patency during severe hypoxia in neonatal rats. We measured dynamic changes in subglottal pressure during normoxic and hypoxic conditions in an arterially perfused brainstem preparation in which we could control gas tensions accurately. Initially, hypoxia $\left(5 \% \mathrm{O}_{2}, 5 \% \mathrm{CO}_{2}\right.$, and $90 \%$ nitrogen) produced an excitatory response in phrenic nerve activity accompanied by augmentation of both inspiratory-related glottal dilation and postinspiratory glottal constriction. Later, during the early stages of hypoxia-induced respiratory depression and initiation of gasp-
\end{abstract}

\section{ABSTRACT}

ing, we observed a massive reduction of the respiratory modulation of glottal resistance. In most preparations, this was transient and replaced by a paradoxic inspiratory-related glottal constriction. We propose that during severe hypoxia in the in situ preparation, paradoxic inspiratory glottal constriction can be observed during gasping, and this may impair ventilation despite the persistence of rhythmic contractions of the respiratory muscles. The latter is of clinical interest, because this may relate to the finding of cot death victims who died as a result of upper airway obstruction but without apparent apnea or rebreathing. (Pediatr Res 58: 193-198, 2005)

PN, phrenic nerve

\section{Abbreviations}

PNA, phrenic nerve activity

SGP, subglottal pressure
Classically, hypoxia triggers a biphasic respiratory response that consists of an initial augmentation, then a marked depression of ventilation; this is followed by gasping until death (1). The characteristics of the hypoxic ventilatory response undergoes postnatal maturation (2). For example, in neonates, the early increase in respiration in response to hypoxia is less pronounced (2). However, neonates have a remarkable tolerance to hypoxia and robust mechanisms for autoresuscitation after hypoxia-induced respiratory depression (3-5). A major component for successful autoresuscitation is gasping $(4,5)$, a breathing pattern triggered during marked hypoxia or anoxia. Because hypoxia is a common complication in the human neonate (3), a knowledge of the precise physiologic response to hypoxia is of clinical interest.

Gasping is a unique breathing pattern defined as having a decrementing inspiratory discharge (as opposed to the augmenting pattern of eupnea) and a synchronization of inspira-

Received August 4, 2004; accepted January 14, 2005.

Correspondence: Mathias Dutschmann, M.D., Department of Physiology, University of Göttingen, Humboldtallee 23, 37076 Göttingen, Germany; e-mail: matt@ukps.gwdg.de.

This study was supported by the British Heart Foundation (BS/93003), the Deutsche Forschungsgemeinschaft (Du 338-1/1), and the SFB 430.

DOI: 10.1203/01.PDR.0000169968.07488.AD tory discharges in cranial (vagal, hypoglossal) and phrenic motor outflows (6-8). In eupnea, there is a highly coordinated initiation of the motor pattern sequence of upper airway and respiratory pump motor activity such that the latter is temporally delayed so that decrease of upper airway resistance can precede the onset of lung inflation. This being the case, the respiratory cycle can be divided into three phases: inspiration, postinspiration, and expiration. We believe expression of all three phases is an important characteristic of the eupneic (normal) breathing pattern at rest (9). The postinspiratory phase provides a motor drive that causes a transient contraction of glottal constrictor muscles. Because of the high recoil property of the lung in the newborn, this phase is of crucial importance. It both maintains functional residual capacity to prevent atelectasis during expiration and stalls expiratory airflow, thereby providing more time for pulmonary gas exchange $(9,10)$. This may be critical in smaller species (e.g. the rat) with a high ventilatory frequency. However, the question as to what happens to glottal constrictor activity early in expiration during hypoxia-induced gasping is raised. On the basis of previous reports, this seems to be inconsistent (11-13) and may relate to the severity and the length of exposure to hypoxia/anoxia. In one study, gasping during severe hypoxia was reported to be 
accompanied by a massive reduction or total abolishment of postinspiratory motor activity destined for the upper airway $(11,14)$. Recent studies showed that an obvious absence of postinspiratory motor activity does not necessarily mean a cessation of the activity of postinspiratory motor neurones (15-17). We found that postinspiratory neurones (including adductor motor neurones) discharged earlier in the respiratory cycle during the inspiratory phase. This was demonstrated by pharmacologic blockade of inhibitory glycinergic neurotransmission, which abolished synaptic inhibition of postinspiratory neurones during the inspiratory phase and revealed an inspiratory depolarization (15-17). Functionally, this had significant effects on respiratory modulation of upper airway resistance. The phase shift of postinspiratory adductor motor discharge into neural inspiration manifested a paradoxic inspiratory glottal constriction $(16,17)$.

In the present study, we investigated the behavior of the upper airway during hypoxic insults in the newborn rat and sought to determine whether, as with glycinergic blockade, prolonged severe hypoxia results in inspiratory efforts against an actively constricted glottis. We used the in situ perfused brainstem preparation because of the technical difficulties of maintaining physiologic viability of newborn rat pups in vivo. This in situ preparation allows fine control of both the arterial pressure and the partial pressure of gases within the circulation as well as being free of anesthesia that is known to depress the respiratory rhythm generator.

\section{METHODS}

The study was approved by the local ethics committee for animal care at the University of Bristol and carried out according to the Animals Act 1986 (Scientific Procedures).

Working heart-brainstem preparation. Wistar rat pups of 2-4 d of age were used $(n=9)$. The in situ arterially perfused preparation used has a well-oxygenated brainstem and normal $\mathrm{pH}$ and generates an eupneic pattern of respiratory motor activity $(18,19)$. Rat pups were anesthetized deeply with halothane. Once respiration was depressed severely and the animal failed to respond to noxious pinch of a toe or the tail, it was transected below the diaphragm. In chilled Ringer's solution gassed with $95 \% \mathrm{O}_{2}$ and $5 \% \mathrm{CO}_{2}$ (carbogen), rats then were decerebrated at the precollicular level under visual control using a binocular microscope. After these initial procedures, which took between 5 and $8 \mathrm{~min}$, halothane anesthesia was discontinued and the preparation was transferred to a recording chamber (custom made). The descending aorta was cannulated and perfused using a peristaltic pump (505S; Watson \& Marlow, Falmouth, UK) via a custom-made catheter with carbogen gassed Ringer's that contained Ficoll (see below) at $31^{\circ} \mathrm{C}$ and at a flow rate of $15-20 \mathrm{~mL} / \mathrm{min}$. The perfusate was filtered and passed through bubble traps (custom made) to remove gas bubbles and dampen pressure pulsations. The perfusate that leaked form the preparation was collected and recirculated after its recarbogenation. Two to $5 \mathrm{~min}$ after the start of perfusion, rhythmic contractions of the diaphragm resumed.

The perfusate contained (in $\mathrm{mM}$ ) $125 \mathrm{NaCl}, 24 \mathrm{NaHCO}_{3}, 2.5 \mathrm{CaCl}_{2}, 5 \mathrm{KCl}$, $1.25 \mathrm{MgSO}_{4}, 1.25 \mathrm{KH}_{2} \mathrm{PO}_{4}, 10$ D-glucose, and Ficoll (1.25\%) to maintain colloid osmotic pressure. All chemicals for Ringer's solution and perfusate were ordered from Sigma Chemical Co.-Aldrich (UK). The osmolarity of the Ringer's solution was $298 \pm 5 \mathrm{mOsmol} / \mathrm{L}$, and on gassing with carbogen, the $\mathrm{pH}$ was $7.35 \pm 0.05$.

Measuring respiratory phrenic nerve activity and subglottal pressure. Respiratory motor activity was recorded from a phrenic nerve (PN; left or right) via a suction electrode. Electrical signals were passed through a headstage and preamplifier, and signals were amplified and filtered $(8 \mathrm{~Hz}-3 \mathrm{kHz}$; Neurolog modules 104 and 125).

Changes in resistance through the larynx were made directly by measuring subglottal pressure (SGP). The trachea below the larynx was cannulated in the direction of the pharynx with a T-shaped catheter. Through one arm of this cannula was passed a constant flow of warmed $\left(31^{\circ} \mathrm{C}\right)$ and humidified carbo- gen gas in the expiratory direction (20). SGP was recorded via the other side of the T-shaped cannula using a pressure transducer (Gould Statham P23XL). The posterior pharyngeal wall just above the larynx was opened to allow the gas to escape. Increases and decreases in SGP were indicative of constriction (adduction) and dilation (abduction), respectively, thereby giving an index of the dynamic changes in upper airway resistance during the respiratory cycle. The rate of airflow through the larynx was individually adjusted for each rat pup until modulation of upper airway resistance was optimized $(20-25 \mathrm{~mL} /$ min). Initially, the air stream that passed through the larynx could cause a depression of breathing (e.g. lengthening of the expiratory interval) and bradycardia, presumably as a result of activation of laryngeal receptors. This response either disappeared spontaneously or was avoided by reducing airflow rate. In all cases, eupnea-like breathing pattern and heart rate returned to control levels.

Experimental protocols. Hypoxia was induced by gassing the perfusate with $5 \% \mathrm{O}_{2}$ and $5 \% \mathrm{CO}_{2}$ (balance nitrogen) for $3 \mathrm{~min} \pm 20 \mathrm{~s}$. Exposure to hypoxia evoked in the PN activity (PNA) a biphasic response that consisted of an initial augmentation of frequency followed by secondary hypoxic depression. The onset of the augmentation of PNA frequency occurred $90 \pm 20 \mathrm{~s}$ after the switch from carbogen to the hypoxic gas mixture (see Figs. $1 A$ and $2 A$ ). We divided the hypoxic depression into two stages defined by the PNA burst frequency: stage I showed a modest depression of $20-40 \%$ of control frequency $0-40 \mathrm{~s}$ after hypoxic augmentation; whereas stage II $10-60 \mathrm{~s}$ later and was characterized by a pronounced depression in frequency (50-80\% of control).

Data analysis. All data were digitized (1401plus CED) and stored on a computer. Respiratory motor nerve activity was integrated off-line (100-ms time constant). Data analysis was performed off-line using CED Spike 2 software (Cambridge Electronic Design, UK).

An index of upper airway resistance was measured from recordings of SGP $(n=9)$. We measured peak SGP values of every respiratory cycle during inspiration, during postinspiration, and at the end of expiration over 10-s periods for control and during the hypoxic challenge. Mean values for these 10 -s periods were determined. To quantify the effect of hypoxia on inspiratory and postinspiratory modulation of glottal resistance, we set the value of SGP at the end of expiration (so-called stage II expiration) as zero. This allowed hypoxia-induced changes in the strength of laryngeal muscle activity to be expressed as a negative value in $\mathrm{mm} \mathrm{Hg}$ for upper airway dilation and positive value $(\mathrm{mm} \mathrm{Hg})$ for upper airway constriction to be expressed relative to end expiration (e.g. zero). In parallel, we measured mean PNA burst frequency, amplitude, and duration in the same 10-s intervals from the raw signal of the PNA. Time-dependent changes in SGP values during hypoxia for single experiments were pooled in line diagrams illustrating the progressive changes in PNA and SGP pattern (see Fig. 3). Because the time from the switch to the hypoxic gas mixture until the onset of the initial hypoxic augmentation varied between animals $(90 \pm 20 \mathrm{~s}$; see "Experimental Protocols"), we took the peak hypoxic augmentation of PNA frequency as a reference point to pool the individual response pattern for further statistical evaluation. To simplify, we restricted the statistical evaluation and data presentation (Fig. 3) to $30 \mathrm{~s}$ before and $90 \mathrm{~s}$ after hypoxic augmentation (i.e. during hypoxic depression) after the pooling. To test significance of the time-dependent changes in SGP and PNA pattern, we used a repeated measure ANOVA followed by a Fisher LSD post hoc test. The tests were a priori restricted to time points representative for control activity ( $0 \mathrm{~s}$,) hypoxic augmentation (30 s), and hypoxic depression stages I (60 s) and II (100 s) after onset of the analyzed hypoxic challenge (total $120 \mathrm{~s}$; see Fig. 3).

Statistical evaluation of all experimental data was performed with Systat 8 software (SPSS, Richmond, CA). All data are presented as mean \pm SEM. A $95 \%$ confidence interval was taken as being significant. Insignificant effects are marked NS in "Results."

\section{RESULTS}

Two types of response pattern to normocapnic hypoxia in neonates. The perfused brainstem preparation of neonatal rats (P2-P4) showed the stereotyped pattern of respiratory response during exposure to a hypoxic/normocapnic gas mixture. The response consisted of an initial augmentation of respiratory frequency followed by depression. In most experiments $(n=6$; group 1), we identified two stages of respiratory depression on the basis of the burst frequency of PNA. Stage I occurred during the first $40 \mathrm{~s}$ after hypoxic augmentation and showed a modest depression of the control frequency $(-20$ to $-40 \%)$, 

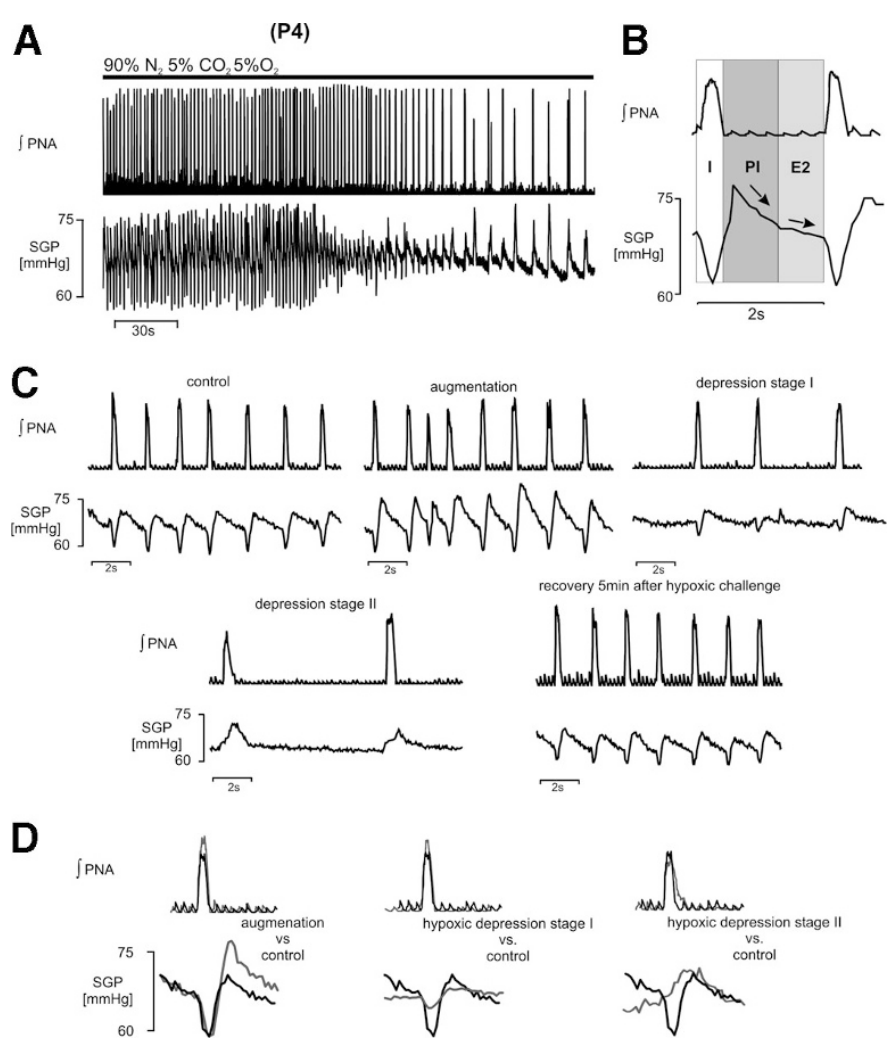

Figure 1. Exposure of six preparations to hypoxia evoked a distinct pattern of response in the central respiratory modulation of upper airway resistance. A representative experiment for this group is shown. (A) The upper trace illustrates an overview of the evoked biphasic hypoxic response in PNA and SGP during exposure to $90 \% \mathrm{~N}_{2}, 5 \% \mathrm{CO}_{2}, 5 \% \mathrm{O}_{2} .(B)$ Magnification of respiratory cycle to illustrate the three-phasic breathing pattern compromising inspiration (I; white area), postinspiration (PI; dark grey area), and late expiration (E2; light grey area). Note that the postinspiration and late expiration can be separated via the slope of the SGP traces (arrows). (C) Selected parts of the hypoxia response such as PNA and SGP during control, respiratory augmentation, and respiratory depression stages I and II are expanded in time. Compared with control, an enhanced postinspiratory upper airway constriction was obvious during hypoxic augmentation of respiratory activity. In contrast, a reduction in upper airway constriction during postinspiration and upper airway opening during PNA was observed during hypoxic depression stage I. Note that during hypoxic depression stage II, upper airway dilation during PNA is now replaced by upper airway constriction indicative of active upper airway constriction during neuronal inspiration. (D) Superimposed traces of integrated PNA and SGP of various stages of the hypoxia response (red lines) compared with control activity. Note that SGP recordings cannot be related to activities of defined muscle groups of the larynx and have to be seen as measurement of overall glottal resistance during a respiratory cycle. $\mathrm{P}$, postnatal age of the rats.

whereas stage II was characterized by a more pronounced depression in frequency $(-50$ to $-80 \%)$ occurring $50-100 \mathrm{~s}$ after hypoxic augmentation. In three experiments after the initial augmentation, the PN burst frequency showed no distinguishable stages and remained constant during hypoxic depression (group 2 rats).

Time-dependent effects of hypoxia on upper airway resistance and PNA. Under control conditions, SGP recordings revealed in all experiments that during neural inspiration (i.e. coincident with PN discharge), SGP decreased corresponding
A

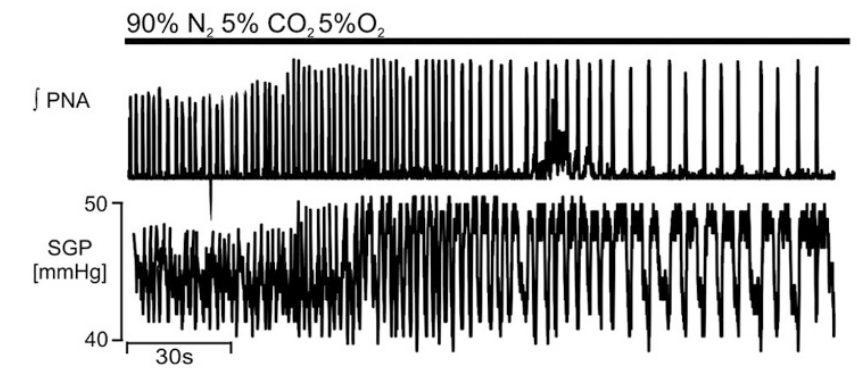

B
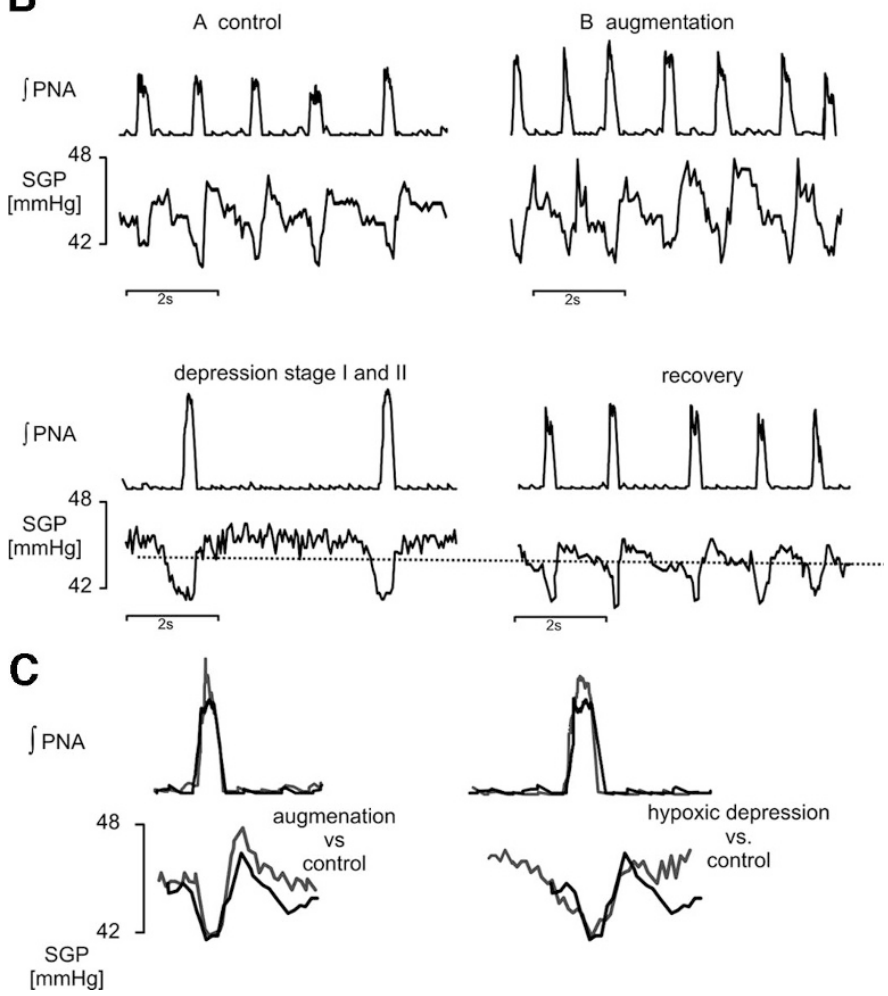

Figure 2. Exposure to hypoxia in three preparations evoked a different pattern of response on the central respiratory modulation of upper airway resistance compared with Fig. 1. (A) The upper trace illustrates an overview of the evoked biphasic hypoxic response in PNA and SGP during exposure of the preparation to $90 \% \mathrm{~N}_{2}, 5 \% \mathrm{CO}_{2}, 5 \% \mathrm{O}_{2}$. (B) Selected parts of the hypoxia response such as PNA and SGP during control, respiratory augmentation, and respiratory depression stages I and II are expanded in time. Compared with control, an enhanced postinspiratory upper airway constriction was found during hypoxic augmentation of respiratory activity. In contrast to the pattern of upper airway modulation shown in Fig. 1, there was no difference in the respiratory modulation of upper airway resistance between hypoxic depression stages I and II. Note that upper airway dilation during PNA was fully preserved and that there was an overall increase in SGP (dotted line) indicative of increased laryngeal tone. $(C)$ Superimposed traces of integrated PNA and SGP of various stages of the hypoxia (red lines) compared with control activity.

to contraction of abductor muscles (i.e. dilation of the vocal cord). Laryngeal constriction occurred immediately after neural inspiration in the postinspiratory phase as seen by a sharp rise in SGP (Figs. 1 and 2). During the latter half of expiration, SGP values slowly returned to a steady state, marking the end of the postinspiratory phase and the start of the expiratory phase (see Fig. 1B). The dynamic changes in SGP and PNA were reported separately for two groups of experiments (see above). 
A
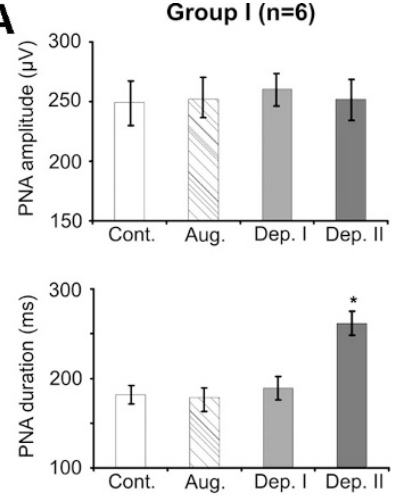

B
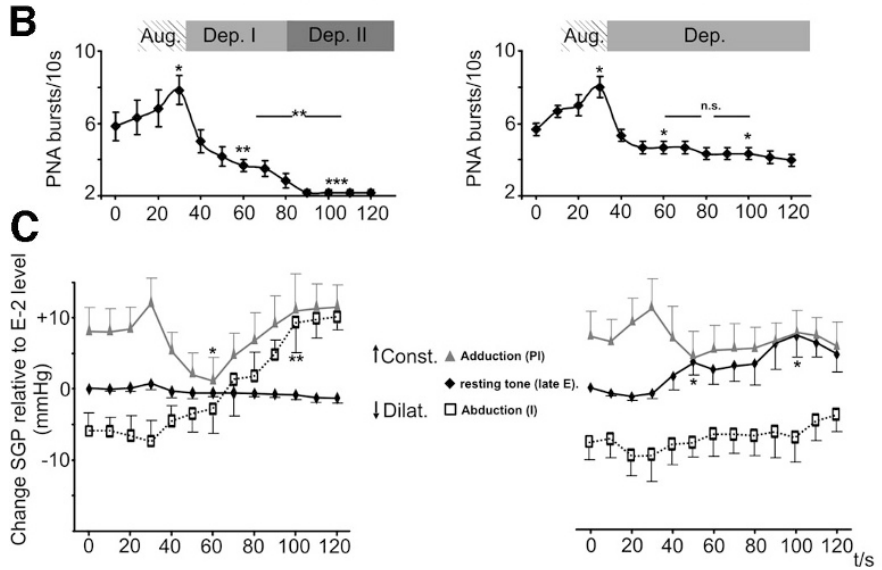

Figure 3. Change in PNA duration and amplitude during various stages of the hypoxic response for the two rat groups analyzed (group 1, $n=6$; group 2, $n=3$ preparations). (B) Mean respiratory frequency during the hypoxic challenge. $(C)$ Time-dependent changes in upper airway resistance during progressive hypoxia for the two types of response observed. The changes of upper airway resistance are expressed as relative changes of SGP compared with the E2 level (mean \pm SEM). Positive values are indicative for upper airway constriction, whereas negative values represent dilation. $\diamond$, SGP values in expiration; $\square$, inspiration; , postinspiration. Tests for significance were restricted to three time points of the hypoxic challenge: after $30 \mathrm{~s}$ (augmentation), $60 \mathrm{~s}$ (depression stage I), and $100 \mathrm{~s}$ (depression stage II). Aug, augmentation; con, control; Dep, depression. * $p<0.05$; ** $p<0.01$; *** $p<$ 0.001 .

Group $1(n=6)$ preparations showed a distinct pattern of changes in PNA and SGP as illustrated in Fig. 1C. During the early stages of hypoxia, these preparations showed an increase in the frequency of PNA from $5.8 \pm 0.8$ to $7.8 \pm 0.8$ bursts $/ 10$ s $(37.3 \pm 4.7 \% ; p<0.01$; Figs. $1 C$ and $D$ and $3 B)$ but no significant changes in amplitude or duration (Fig. $3 A$ ). During the augmentation phase of the response to hypoxia, both inspiratory decrease and postinspiratory increase of glottal resistance were slightly augmented as illustrated in Figs. $1 C$ and $D$ and $3 C$ (e.g. inspiratory SGP: $-6.1 \pm 1.7$ to $-7.2 \pm 1.9$ $\mathrm{mm} \mathrm{Hg}, 11.9 \%$, NS; and postinspiratory SGP: $8.4 \pm 2.6$ to $12.2 \pm 2.7 \mathrm{~mm} \mathrm{Hg}, 39 \%, p<0.05)$. During hypoxic depression stage I, the frequency of PN discharges decreased from 5.8 \pm 0.8 to $3.6 \pm 0.3$ bursts $/ 10 \mathrm{~s}$ compared with control $(-37.9 \%, p<0.01$; Figs. $1 C$ and $D$ and $3 A)$ and evoked a slight increase in PNA amplitude from $252.3 \pm 20.9$ to $266 \pm$ $23.1 \mu \mathrm{V}(9.6 \pm 3.9 \%$, NS; Fig. $3 A$, also see Fig. $1 B$ and $C)$, whereas the amplitude and the duration of PNA remained unchanged (Fig. $3 A$ ). In parallel, we observed a decrease in the activity of both muscle groups of the larynx (inspiratory SGP: $-6.1 \pm 1.7$ to $-2.9 \pm 2.6 \mathrm{~mm} \mathrm{Hg},-51.5 \%$, NS; postinspiratory SGP: $8.4 \pm 2.6$ to $1.5 \pm 2.1 \mathrm{~mm} \mathrm{Hg},-81.7 \%, p<0.05$; Figs. $1 B$ and $C$ and $3 C$ ). Progression of hypoxia into stage II further decreased PNA frequency to $2.3 \pm 0.1$ bursts $/ 10 \mathrm{~s}$ $(-60.3 \%$ compared with control; $p<0.01$; Fig. $3 B)$ and evoked a significant increase in PNA duration (179.6 \pm 9.8 to $267 \pm 13.8 \mathrm{~ms} ; p<0.05)$, whereas the amplitude remained unchanged $(252.3 \pm 20.9$ to $253 \pm 24 \mu \mathrm{V}$, 9.6, NS; Fig. $1 B$ and $C$ and $3 A$ ). During this stage of hypoxia, postinspiratory adductor activity was enhanced compared with control (8.4 \pm 2.6 versus $11.5 \pm 4.4 \mathrm{~mm} \mathrm{Hg}, 36 \%$, NS), but inspiratory decrease in glottal resistance was completely abolished and was now replaced by glottal constriction during the inspiratory phase (e.g. from $-6.1 \pm 1.7$ to $9.3 \pm 3.4 \mathrm{~mm} \mathrm{Hg} ; p<0.01$ ). The latter is clearly indicated by the shift from negative to positive SGP values during PNA as seen in Fig. $3 C$. Finally, the peak SGP level during the stage II expiration phase did not change significantly during the entire period of the hypoxic challenge, suggesting no overall change in resistance during the latter part of expiration (see diamond symbols of Fig. 3C). In all cases, the eupnea-like modulation of the upper airway recovered 4-8 min after the end of the hypoxic challenge (see Fig 1C).

In group 2 rats $(n=3)$, the analysis of PNA and SGP revealed a different type of response within the upper airway to the hypoxic challenge, which is shown in Fig. 2. The initial augmentation part of PNA and SGP response to hypoxia was comparable to group 1 (see Fig. $2 B$ and $C$ ) and was characterized by an increase in PNA frequency from $5.6 \pm 0.3$ to $8 \pm$ 0.7 bursts $/ 10$ s $(42.9 \% ; p<0.05$; Fig. $3 B)$ accompanied by a clear increase in PNA amplitude from $169.8 \pm 19.1$ to $261 \pm$ $9.6 \mu \mathrm{V}(49.4 \% ; p<0.05)$ but no significant change in PNA duration (from $286 \pm 20.6$ to $239.6 \pm 32.9 \mathrm{~ms}$; Fig. $3 A$ ). At this time, the activity of both laryngeal abductors and adductors was changed $(-7.5 \pm 2.6$ to $-9.5 \pm 4 \mathrm{~mm} \mathrm{Hg}, 26 \%$, NS; $8.5 \pm 3.2$ to $11.8 \pm 4.2 \mathrm{~mm} \mathrm{Hg}, 39 \%$, NS; Fig. $3 C$ ). The single-stage hypoxic depression was characterized by a stable PN firing but of reduced frequency compared with control that persisted ( $5.6 \pm 0.3$ to $4.3 \pm 0.3$ bursts $/ 10 \mathrm{~s} ; p<0.05$; Figs. $2 B$ and $C$ and $3 B$ ). During this part of the hypoxic response, the PNA amplitude remained elevated (from $169.8 \pm 19.1$ to 238.2 $\pm 25.7 \mu \mathrm{V}, \mathrm{NS}$ ), and its duration was decreased further from $286 \pm 20.6$ to $219 \pm 7.2 \mathrm{~ms}(p<0.05$; Fig. $3 A)$. SGP recording during the hypoxic depression revealed two timedependent responses: first, there was a modest depression of laryngeal adductor activity at the beginning of hypoxic depression $(8.5 \pm 3.2$ to $5.4 \pm 3.6 \mathrm{~mm} \mathrm{Hg}$, NS). Second, with continued hypoxia, the postinspiratory SGP returned to control levels $(8.2 \pm 0.4 \mathrm{~mm} \mathrm{Hg})$, but throughout hypoxia, decrease in glottal resistance persisted during neural inspiration (Fig. 2C), which was in contrast to the increase in glottal resistance seen in group 1 rats (Fig. 1C). Also, in contrast to group 1 rats, we observed a progressive change of SGP values during the late expiratory phase (Fig. $3 C$ ). These values were increased immediately after the onset of hypoxic depression (0 to $3.1 \pm 1.8$ $\mathrm{mm} \mathrm{Hg} ; p<0.05)$ and further increased to $8.5 \pm 3.5 \mathrm{~mm} \mathrm{Hg}$ $(p<0.05)$ in later stages of the hypoxic depression. Under this 
condition, SGP values showed no difference throughout the entire expiratory interval (compare $8.2 \pm 0.4 \mathrm{~mm} \mathrm{Hg}$ during postinspiration versus $8.5 \pm 3.5 \mathrm{~mm} \mathrm{Hg}$ during late expiration). Five to 6 min after the hypoxic challenge, the eupnea-like modulation of upper airway resistance returned (Fig. 2B).

\section{DISCUSSION}

Our study revealed two different response types during hypoxic depression of ventilation in the neonatal rat. Each response type was associated with a different pattern in the modulation of upper airway resistance after prolonged exposure to hypoxia. The predominant pattern (66\% of animals) was associated with a paradoxic inspiratory-related glottal closure, whereas the second pattern was characterized by 1) an overall increase in glottal resistance but 2) maintenance of inspiratory glottal dilation.

Technical considerations. The perfused brainstem preparation of the neonatal rat provides well-controlled experimental conditions to study the physiologic and pathophysiologic kinesiologic behavior of respiratory network function. A major advantage is that the roller-pump-controlled perfusion system of brain tissue prevents the secondary effects of a failing cardiovascular system on respiratory network activity during exposure to hypoxia. In contrast, prolonged hypoxia in vivo would cause peripheral vasodilation and inadequate brain perfusion in neonates, thereby compromising their viability.

Although the respiratory activity generated by the in situ arterially perfused brainstem preparation largely reflects a eupnea-like pattern $(6,18)$, it cannot be fully equated to eupnea in vivo. A main difference is the significantly lower breathing frequency compared with the in vivo situation and the absence of phasic lung inflation (21). The last two may influence the effects on the postinspiratory glottal constrictor activity revealed in the SGP recordings herein. Finally, it has to be clarified that SGP recordings cannot be related to activities of defined muscle groups of the larynx and have to be seen as measurement of overall glottal resistance during a respiratory cycle.

Resistance to hypoxia and laryngeal response patterns. A major difference between neonatal and adult rat responses to hypoxia is the persistence of gasping PNA in the newborns. In adults, there are a limited number of gasps before terminal apnea commences (22). As seen here, this is clearly not the case in neonatal rats. However, gasping frequency could decrease toward the end of a hypoxia challenge, and this may reflect brainstem anoxia. That our preparations from neonatal rat showed a persistence of rhythmic activity under prolonged hypoxia is in accordance with previous reports in neonatal rats (23). The ability of respiratory neurones to generate rhythmic activity using anaerobic metabolism is a specific feature of neonates (23), because in mature mammals, rhythmic respiratory drive potentials cease in medullary respiratory neurones $(24,25)$ in the absence of oxygen. These characteristics of neonatal respiratory neurones undoubtedly contribute to the remarkable high tolerance to hypoxia, assistance in the maintenance of rhythmic activity, and successful autoresuscitation of newborns (26).
A main result of the present study was the heterogeneity in the response pattern of modulation of upper airway resistance during severe hypoxia. This was evidenced by the majority showing a paradoxic inspiratory-related glottal constriction, whereas others maintained inspiratory-related glottal opening with an overall increase in laryngeal tone. Regarding the former, it is likely that because of the experimental design, we are underestimating the inspiratory-related increase in glottal resistance. With a steady stream of gas flowing in the expiratory direction, this itself will keep the glottis open. In a real-life scenario, negative pulmonary pressure behind an obstructing glottis during inspiration is likely to close the glottis. What remains unclear is why there are two patterns of response, but small differences in brain tissue $\mathrm{Po}_{2}$ could account for it. Evidence for this assumption is arising from the different gasping patterns identified for the two groups. Group 1 showed a prolongation of the PNA compared with control, whereas group 2 showed a decrease. These variations might reflect different operational states of the respiratory network during progressive withdrawal of oxygen.

Inspiratory-related glottal closure: a role for glycinergic inhibition. It is a widely accepted hypothesis that under eupnea, the respiratory pattern and rhythm are generated by network oscillations that depend on reciprocal synaptic inhibition (27). During hypoxia or ischemia, the synaptic respiratory network activity reconfigures to generate the terminal activity pattern of gasping (22). This transformation is likely to include disturbances of ion homeostasis within the brain (e.g. increased extracellular potassium ion concentration), membrane depolarization, and failure of chloride ion-mediated synaptic inhibition provided by glycinergic and GABAergic neurones of the respiratory network $(15-17,23,25,28)$. In particular, glycinergic inhibition was demonstrated to be essential for the coordination of respiratory motor output to cranial (e.g. laryngeal airway resistance muscles) and spinal (e.g. pump muscles) targets. Pharmacologic blockade of glycine receptors in the neonatal or adult respiratory network caused a paradoxic inspiratory glottal constriction, although rhythmic network activity persisted $(16,17)$. This result parallels the upper airway response to severe hypoxia demonstrated in the present study. It very likely that during hypoxia in the present experiments, synaptic inhibition failed (25) and produced a pattern of inspiratory glottal constriction.

A physiologic role for different patterns of laryngeal modulation during hypoxia. Both forms of gasping showed a full recovery to the eupnea-like modulation of upper airway resistance after re-oxygenation. This raises the question as to the physiologic roles of both forms of gasping.

In group 2 rats, the overall increase of glottal resistance observed during hypoxic gasping would assist in maintaining the residual capacity of the lungs and prevent atelectasis. This coupled with a forced inspiratory effort would provide adequate ventilation (12). Thus, this pattern can be seen as an attempt of the respiratory network to overcome hypoxia and may play a crucial role for autoresuscitation $(12,26)$. However, in group 1 rats, the more frequently found paradoxic inspiratory upper airway constriction would clearly impair any attempt for re-oxygenation. Nevertheless, the persistence of the 
rhythm might be important for successful auto resuscitation of network activity. A possible physiologic meaning could be that this inspiratory glottal closure prevents aspiration of fluids that accumulate in the upper airways.

Clinical implications. Obstructive apnea and hypopnea are common clinical problems in human infants and are often related to sleep $(29,30)$. Although these complications are often attributed to anatomic abnormalities of the upper airway, there is growing evidence that pathologic disturbance in the neural control of breathing has major influence on the pathogenesis (31). As discussed, a failure of glycinergic synaptic inhibition might be one of the main factors leading to inspiratory upper airway obstruction. It is interesting that developmental studies on glycine receptors revealed a switch from a neonatal to an adult isoform between 17 and $22 \mathrm{~d}$ of life in the rat (32). A similar switch of glycine receptor isoforms might generate a vulnerable period during the early postnatal development of humans. It is interesting that it was reported that cot-death victims died as a result of upper airway obstruction but without apparent respiratory arrest or rebreathing (33). Whether cotdeath victims had decreased level of glycine receptors in the respiratory network medulla is presently unknown.

A phylogenetic origin for inspiratory upper airway modulation during hypoxia. To our knowledge, the only respiratoryrelated activity correlating with inspiratory glottal closure is the hiccup (34). It is interesting that the hiccup is apparently one of the first respiratory-related activities reported for the mammalian fetus. According to the theory that evolution is reflected by the gestational stages of the embryo, this might suggest that the hiccup has an old origin. Indeed, in a recent article by Strauss et al. (34) it was reviewed that the hiccup potentially reflects the most archaic form of breathing - this being gill ventilation, which is characterized by synchronous activation of inspiratory muscles during glottal closure. Therefore, a progressing failure of respiratory network function during hypoxia may potentially reveal ancient forms of breathing that were not designed for air breathing.

\section{REFERENCES}

1. Powell FL, Milsom WK, Mitchell GS 1998 Time domains of the hypoxic ventilatory response. Respir Physiol 112:123-134

2. Eden GJ, Hanson MA 1987 Maturation of the respiratory response to acute hypoxia in the newborn rat. J Physiol 392:1-9

3. Martin RJ, DiFiore JM, Jana L, Davis RL, Miller MJ, Coles SK, Dick TE 1998 Persistence of the biphasic ventilatory response to hypoxia in preterm infants. J Pediatr 132:960-964

4. Sanocka UM, Donnelly DF, Haddad GG 1992 Autoresuscitation: a survival mechanism in piglets. J Appl Physiol 73:749-753

5. Gozal D, Gozal E, Reeves SR, Lipton AJ 2002 Gasping and autoresuscitation in the developing rat: effect of antecedent intermittent hypoxia. J Appl Physiol 92:11411144
6. St John WM, Paton JF 2003 Defining eupnea. Respir Physiol Neurobiol 139:97-103

7. Richter DW 2003 Commentary on eupneic breathing patterns and gasping. Respir Physiol Neurobiol 139:121-130

8. Duffin J 2003 A commentary on eupnoea and gasping. Respir Physiol Neurobiol 139:105-111

9. Dutschmann M, Paton JF 2002 Inhibitory synaptic mechanisms regulating upper airway patency. Respir Physiol Neurobiol 131:57-63

10. Bartlett DJ 1986 Upper airway motor systems. In: Cherniack NS, Widdicombe JG (eds) Handbook for Physiology, Sec 3, The Respiratory System, Vol. II. American Physiological Society, Bethesda, pp 223-245

11. St John WM, Zhou D, Fregosi RF 1989 Expiratory neural activities in gasping. J Appl Physiol 66:223-231

12. Thuot F, Lemaire D, Dorion D, Letourneau P, Praud JP 2001 Active glottal closure during anoxic gasping in lambs. Respir Physiol 128:205-218

13. Hutchison AA, Burchfield DJ, Wozniak JA, Mohrman SJ 2002 Laryngeal muscle activities with cerebral hypoxia-ischemia in newborn lambs. Am J Respir Crit Care Med 166:85-91

14. St John WM, Rybak IA, Paton JF 2002 Potential switch from eupnea to fictive gasping after blockade of glycine transmission and potassium channels. Am J Physiol 283:R721-R731

15. Büsselberg D, Bischoff AM, Paton JF, Richter DW 2001 Loss of glycinergic inhibition reveals two modes of respiratory rhythm generation. Pflugers Arch 441:444-449

16. Dutschmann M, Paton JF 2002 Glycinergic inhibition is essential to co-ordinate cranial and spinal motor outputs of the central respiratory network in the neonatal rat. J Physiol 543:643-653

17. Dutschmann M, Paton JFR 2002 Trigeminal reflux regulation of the glottis depends on central glycinergic inhibition in the rat. Am J Physiol 282:R999-R1005

18. Dutschmann M, Wilson RJ, Paton JF 2000 Respiratory activity in neonatal rats. Auton Neurosci 84:19-29

19. Wilson RJ, Remmers JE, Paton JF 2001 Brain stem $\mathrm{PO}(2)$ and $\mathrm{pH}$ of the working heart-brain stem preparation during vascular perfusion with aqueous medium. Am J Physiol 281:R528-R538

20. Paton JF, Li YW, Kasparov S 1999 Reflex response and convergence of pharyngoesophageal and peripheral chemoreceptors in the nucleus of the solitary tract. Neuroscience 93:143-154

21. Mortola JP, Magnante D, Saetta M 1985 Expiratory pattern of newborn mammals. J Appl Physiol 58:528-533

22. St-John WM 1998 Neurogenesis of patterns of automatic ventilatory activity. Prog Neurobiol 56:97-117

23. Ballanyi K, Onimaru H, Homma I 1999 Respiratory network function in the isolated brainstem-spinal cord of newborn rats. Prog Neurobiol 59:583-634

24. Richter DW, Bischoff A, Anders K, Bellingham M, Windhorst U 1991 Response of the medullary respiratory network of the cat to hypoxia. J Physiol 443:231-256

25. Schmidt C, Bellingham MC, Richter DW 1995 Adenosinergic modulation of respiratory neurones and hypoxic responses in the anaesthetized cat. J Physiol (Lond) 483:769-781

26. Fewell JE, Smith FG, Ng VK, Wong VH, Wang Y 2000 Postnatal age influences the ability of rats to autoresuscitate from hypoxic-induced apnea. Am J Physiol 279:R39-R46

27. Richter DW, Spyer KM 2001 Studying rhythmogenesis of breathing: comparison of in vivo and in vitro models. Trends Neurosci 24:464-472

28. Rybak IA, Shevtsova NA, St-John WM, Paton JF, Pierrefiche O 2003 Endogenous rhythm generation in the pre-Botzinger complex and ionic currents: modelling and in vitro studies. Eur J Neurosci 18:239-257

29. Gaultier C 2001 Abnormalities of the chemical control of breathing: clinical correlates in infants and children. Pediatr Pulmonol Suppl 23:114-117

30. Gislason T, Benediktsdottir B 1995 Snoring, apneic episodes, and nocturnal hypoxemia among children 6 months to 6 years old. An epidemiologic study of lower limit of prevalence. Chest 107:963-966

31. Fregosi RF, Quan SF, Jackson AC, Kaemingk KL, Morgan WJ, Goodwin JL, Reeder JC, Cabrera RK, Antonio E 2004 Ventilatory drive and the apnea-hypopnea index in six-to-twelve year old children. BMC Pulm Med 4:4

32. Kling C, Koch M, Saul B, Becker CM 1997 The frameshift mutation oscillator (Glra1(spd-ot)) produces a complete loss of glycine receptor $\alpha 1$-polypeptide in mouse central nervous system. Neuroscience 78:411-417

33. Poets CF, Meny RG, Chobanian MR, Bonofiglo RE 1999 Gasping and other cardiorespiratory patterns during sudden infant deaths. Pediatr Res 45:350-354

34. Straus C, Vasilakos K, Wilson RJ, Oshima T, Zelter M, Derenne JP, Similowski T, Whitelaw WA 2003 A phylogenetic hypothesis for the origin of hiccough. Bioessays 25:182-188 